\title{
Evaluation of the frequency of left renal vein variations in computed tomography and its relationship with cancer development
}

\author{
T.Y. Kuzan1, B.N. Kuzan², T.A. Telli³, D. Tüney² \\ 'Department of Radiology, Sancaktepe Sehit Prof. Dr. Ilhan Varank Training and Research Hospital, Istanbul, Turkey \\ 2Department of Radiology, Marmara University School of Medicine, Istanbul, Turkey \\ 3Division of Medical Oncology, Department of Internal Medicine, Marmara University School of Medicine, Istanbul, Turkey
}

[Received: 24 October 2019; Accepted: 28 November 2019]

\begin{abstract}
Background: Left renal vein ( $L R V)$ variations occur in $0.8-10.2 \%$ of the population. The most common $L R V$ variations are retroaortic left renal vein (RLRV) and circumaortic left renal vein (CLRV). The purpose of this study is to determine the frequency of $L R V$ variations in a large series on computed tomography (CT) and to investigate the association between LRV and malignancy development.

Materials and methods: Between January 2015 and January 2017, an abdominal CT examination of 12,341 (5505 female, 6836 male) patients was evaluated retrospectively in this study. Patients' clinical and demographic data were recorded using the Hospital Information System.

Results: Left renal vein variations were detected in 314 (2.54\%) of the 12,341 patients within the study. Of the 314 cases found to have LRV variations, 227 (1.84\%) had RLRV, and 87 (0.70\%) had CLRV. There was no statistical difference in total $L R V$ variations ( $p=0.083$ ) and CLRV variation ( $p=0.96)$ groups in terms of gender. However, the RLRV variation was found to be 1.32 times higher in males than in females ( $p=0.039)$. Of the 314 patients with LRV variations, 73 (23.2\%) had any sort of concomitant malignancy.

Conclusions: A high incidence of malignancy was detected in patients with LRV variations. Of the $L R V$ variations, $R L R V$ variation is more common than CLRV variation. The presence of total $L R V$ variations and CLRV variations is not associated with gender; whereas the presence of RLRV variation is more common in males. (Folia Morphol 2020; 79, 4: 793-798)
\end{abstract}

Key words: renal vein, variation, abnormality, malignancy, computed tomography, circumaortic, retroaortic

\section{INTRODUCTION}

Inferior vena cava (IVC) and renal vein variations originate from the anomalies in the anastomoses of the three paired veins, including subcardinal, supracardinal and postcardinal veins, during embryonic development, and they may have numerous forms $[8,14]$. Normally, a single left renal vein (LRV) drains into the IVC by crossing anteriorly over the abdominal aorta in the majority of cases. However, the anomalies arising in the LRV during the complex embryogenesis lead to the formation of variations $[2,10,16]$.

Left renal vein variations occur in $0.8-10.2 \%$ of the population, although it varies depending on the research method (Table 1). The most common LRV

Address for correspondence: T.Y. Kuzan MD, Sancaktepe Sehit Prof. Dr. Ilhan Varank Training and Research Hospital, Department of Radiology, 34785 Istanbul, Turkey, tel: +90216-6063300, fax: +90216-6063395, e-mail: tykuzan@gmail.com

Study Centre: Marmara University Pendik Training and Research Hospital, Istanbul 
Table 1. Frequency of left renal vein variations in studies using different methods

\begin{tabular}{lccccc}
\hline Study (year) [Ref.] & Method & Sample size & RLRV & CLRV & Total LRV \\
\hline 1. (2008) [9] & CT & 153 & $0.7 \%$ & $1.3 \%$ & $2 \%$ \\
2. (1982) [13] & CT & 433 & $1.8 \%$ & $4.4 \%$ & $6.2 \%$ \\
3. (2016) [1] & PET-CT & 222 & $2.70 \%$ & $3.15 \%$ & $5.85 \%$ \\
4. (2013) [5] & CT & 1204 & $3.1 \%$ & $2.1 \%$ & $5.2 \%$ \\
5. (2007) [10] & CT & 1120 & $4.7 \%$ & $5.5 \%$ & $10.2 \%$ \\
6. (2012) [6] & MRI & 2644 & $1.66 \%$ & $1.02 \%$ & $2.68 \%$ \\
7. (2017) [7] & MRI & 3000 & $2.1 \%$ & $0.5 \%$ & $2.6 \%$ \\
8. (2017) [17] & Autopsy & 550 & $4.2 \%$ & $3.8 \%$ & $8.0 \%$ \\
9. (2014) [3] & CT & 746 & $7.4 \%$ & $2.4 \%$ & $9.8 \%$ \\
10. (2015) [19] & CT & 1452 & $2.1 \%$ & $2.1 \%$ & $4.2 \%$ \\
11. (2004) [18] & CT & 984 & $2.3 \%$ & $0.9 \%$ & $3.2 \%$ \\
12. (1999) [16] & Autopsy and in-vivo & 1008 & $0.5 \%$ & $0.3 \%$ & $0.8 \%$ \\
13. (2016) [4] & CTA & 504 & $4.2 \%$ & $5.2 \%$ & $9.7 \%$ \\
\hline
\end{tabular}

CLRV — circumaortic left renal vein; CT — computed tomography; CTA — computed tomography angiography; LRV — left renal vein; MRI — magnetic resonance Imaging; PET-CT — positron emission tomography-computed tomography; RLRV — retroaortic left renal vein

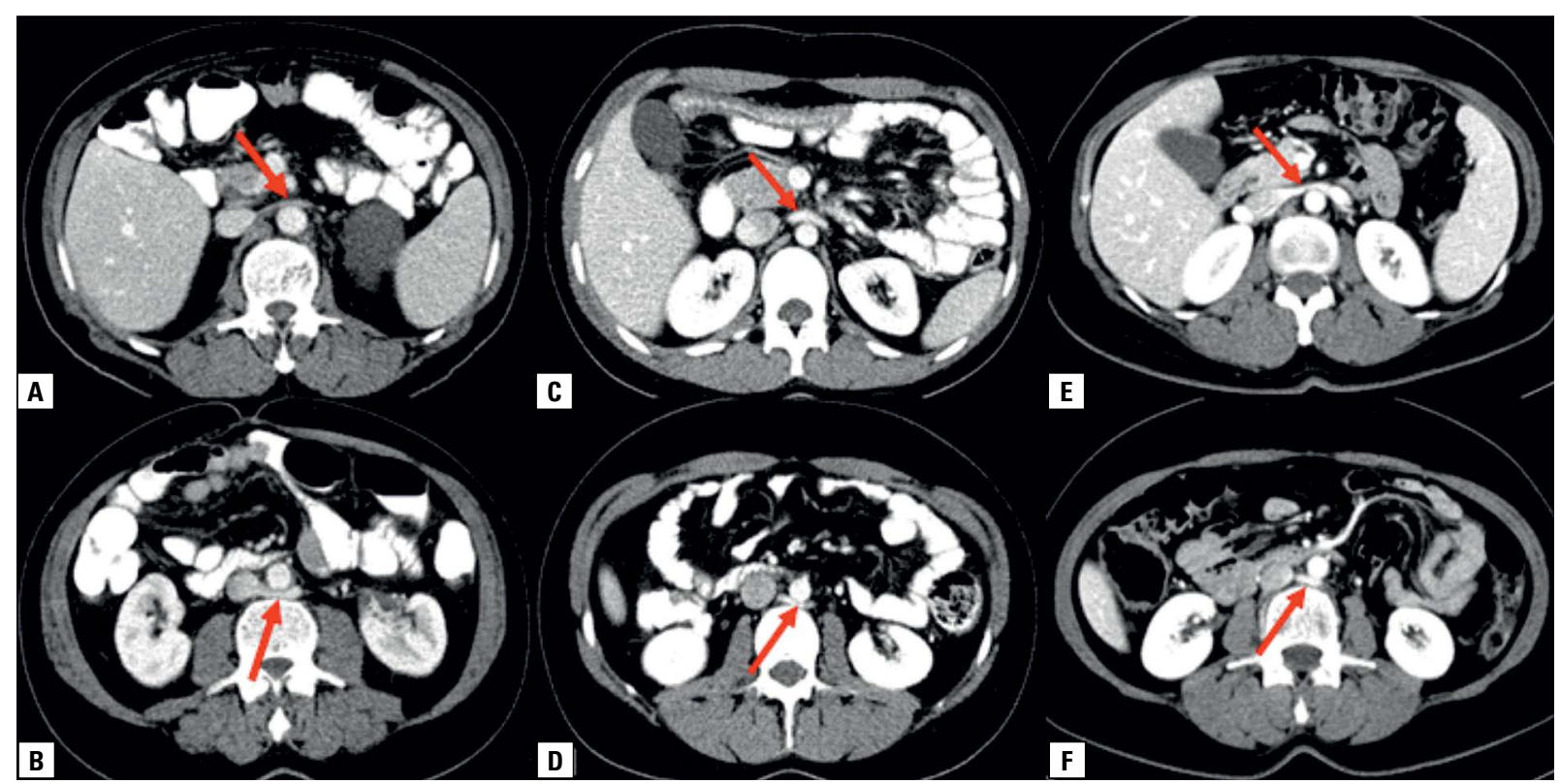

Figure 1. Circumaortic left renal veins in a 66-year-old male patient (A, B) with colon cancer, a 20-year-old male patient (C, D) without concomitant malignancy and a 56 -year-old female patient (E, F) with non-Hodgkin lymphoma. Contrast-enhanced computed tomography scans show the left renal veins coursing anterior (red arrows in panels $A, C, E$ ) and posterior (red arrows in panels $B, D, F$ ) to the aorta.

variations are retroaortic left renal vein (RLRV) and circumaortic left renal vein (CLRV) (Figs. 1, 2). With recent developments in imaging techniques and frequent use of imaging modalities, these abdominal vein variations are identified more frequently during routine examinations. Although it is known that the detection of LRV variations is clinically important for both surgical and diagnostic reasons, the relationship between these variations and malignancy is not known $[7,10,13,16,19]$.

In this study, we aim to determine the frequency of LRV variations in a large series on computed tomography (CT) and to investigate the association between LRV and malignancy development. 


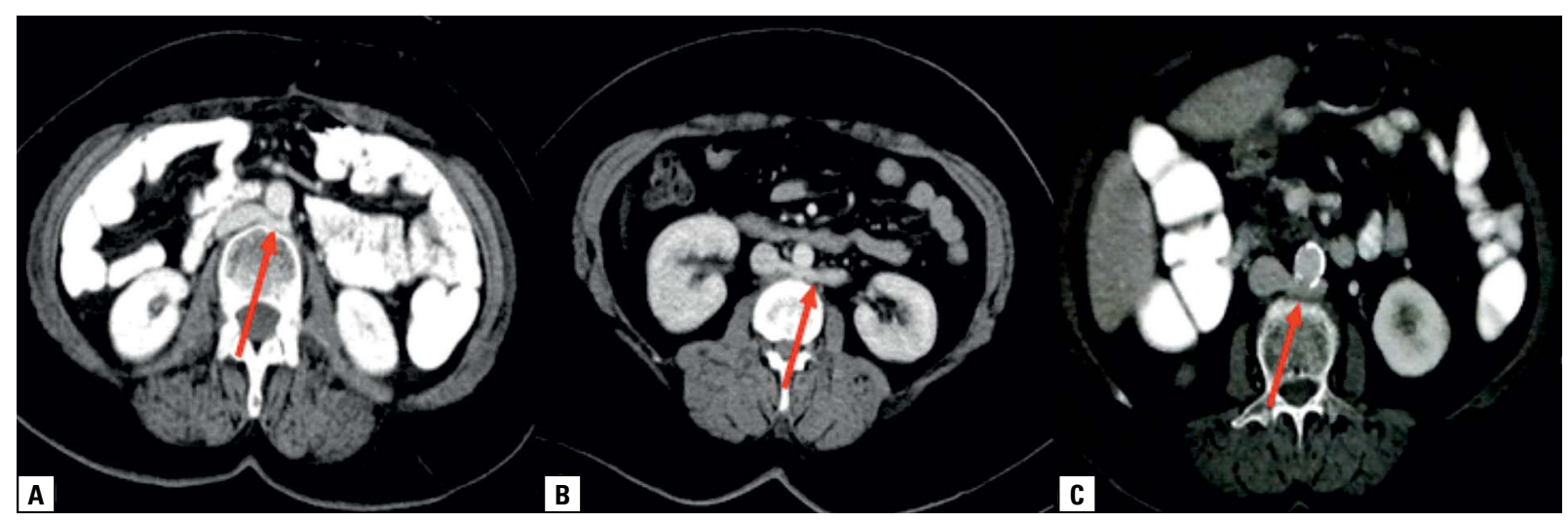

Figure 2. Retroaortic left renal veins in a 62-year-old female patient (A) with colon cancer, a 57-year-old male patient (B) without concomitant malignancy and a 79-year-old female patient (C) with non-Hodgkin lymphoma. Contrast-enhanced computed tomography scans show the left renal veins (red arrows in panels $A, B, C$ ) coursing posterior to the aorta.

\section{MATERIALS AND METHODS}

\section{Patient characteristics}

Approval was obtained from the Marmara University Ethics Committee (No: 09.2018.347) before the study began. The clinical and demographic data of cases were obtained from the hospital information management system and patient files.

The patients abdominal CTs, which were taken for any reason during the 2-year period between January 2015 and January 2017 in Marmara University Pendik Training and Research Hospital, were retrospectively reviewed. Patients whose vascular structures could not be evaluated due to previous abdominal surgery or intraabdominal mass were not included in the study. Only one abdominal CT examination per patient of those who has had multiple CT examinations was included in the study. In total, abdominal CTs of 12,341 patients, which were evaluated by a radiologist with 20 years of abdominal radiology experience, were included in the study.

\section{CT protocol and image interpretation}

Examinations were carried out with 256-slice CT scanner or 16-slice CT scanner devices. The obtained axial images were loaded into a Picture Archiving Communication System workstation and the imaging findings were evaluated using the axial slices and, if necessary, the reformatted multiplanar reconstruction images. The image analysis was performed by the same radiologist using consecutive axial CT images. The course of the LRV was shown uninterruptedly from the kidney hilus to the IVC. An LRV that drains into the IVC by crossing anteriorly over the aorta is accepted to be normal, whereas an LRV draining into the IVC by passing posteriorly over the aorta is defined as RLRV. An LRV complex that drains into the IVC by passing anteriorly and posteriorly around the aorta and forming a venous circle was defined as CLRV.

\section{Statistical analysis}

Descriptive analysis was carried out for the case characteristics. The continuous variables were reported as mean \pm standard deviation. The rates were expressed in numbers and corresponding percentages. The percentages are indicated by confidence intervals (Cls). The data obtained were analysed using the SPSS 17.0 statistical package programme (SPSS Inc., Chicago, IL, USA), and the statistical analysis was assessed at a significance level of $p<0.05$. The correlations of the categorical variables were determined using the $\chi^{2}$ test. The male and female cases were compared using probability rates and $\mathrm{Cls}$.

\section{RESULTS}

Left renal vein variations were detected in 314 $(2.54 \%)$ of the 12,341 cases within the study. Of these cases, 5505 (44.6\%) were female, 6836 $(55.4 \%)$ were male and the median age was 46.5 (distribution: 4-88 years). Of the 314 cases found to have LRV variations, 227 (1.84\%) had RLRV, and $87(0.70 \%)$ had CLRV.

In females, the total number of cases with LRV variations, RLRV variation and CLRV variation (with corresponding percentages) was 125 (2.27\%), 86 $(1.56 \%)$ and $39(0.71 \%)$, respectively. In males, the total number of cases with LRV variations, RLRV variation and CLRV variation (with corresponding percentages) was 189 (2.76\%), 141 (2.06\%) and 48 (0.70\%), 
Table 2. The frequencies and distribution of left renal vein (LRV) variations in cases

\begin{tabular}{lccc}
\hline & $\begin{array}{c}\text { Total }(\mathbf{n}=12341) \\
\text { No. of patients }(\%)\end{array}$ & $\begin{array}{c}\text { Females }(\mathbf{n}=\mathbf{5 5 0 5}) \\
\text { No. of patients }(\%)\end{array}$ & $\begin{array}{c}\text { Males }(\mathbf{n}=\mathbf{6 8 3 6}) \\
\text { No. of patients }(\%)\end{array}$ \\
\hline Retroaortic LRV variations & $227(1.84 \%)$ & $86(1.56 \%)$ & $141(2.06 \%)$ \\
Circumaortic LRV variations & $87(0.70 \%)$ & $39(0.71 \%)$ & $48(0.70 \%)$ \\
Total LRV variations & $314(2.54 \%)$ & $125(2.27 \%)$ & $189(2.76 \%)$ \\
\hline
\end{tabular}

Table 3. The frequencies and distribution of retroaortic (RLRV) and circumaortic (CLRV) left renal vein (LRV) variations

\begin{tabular}{lccc}
\hline \multirow{2}{*}{ Variation } & \multicolumn{2}{c}{ Gender } & Total \\
\cline { 2 - 3 } & Female & Male & \\
\hline CLRV & & & \\
Count & 39 & 48 & 87 \\
\% within CLRV & $44.8 \%$ & $55.2 \%$ & $100.0 \%$ \\
\% within gender & $31.2 \%$ & $25.4 \%$ & $27.7 \%$ \\
\% of total & $12.4 \%$ & $15.3 \%$ & $27.7 \%$ \\
RLRV & & & \\
Count & 86 & 141 & 227 \\
\% within RLRV & $37.9 \%$ & $62.1 \%$ & $100.0 \%$ \\
\% within gender & $68.8 \%$ & $74.6 \%$ & $72.3 \%$ \\
\% of total & $27.4 \%$ & $44.9 \%$ & $72.3 \%$ \\
Total LRV & & & \\
Count & 125 & 189 & 314 \\
\% within LRV & $39.8 \%$ & $60.2 \%$ & $100.0 \%$ \\
\% within gender & $100.0 \%$ & $100.0 \%$ & $100.0 \%$ \\
\% of total & $39.8 \%$ & $60.2 \%$ & $100.0 \%$ \\
\hline
\end{tabular}

respectively. The frequencies and distribution of LRV variations are given in Tables 2, 3.

The correlation of LRV variations with gender was determined using the $\chi^{2}$ test. No statistically significant correlation was found between gender and total LRV variations ( $p=0.083$ ). CLRV variation was found to be approximately equally frequent in both males and females. Likewise, no statistically significant correlation was found between CLRV variation and gender $(p=0.96)$. However, the RLRV variation was found to be 1.32 times higher in males than in females (95\% Cl 1.01-1.72), which was statistically significant ( $p=0.039$ ). Thus, the RLRV variation was determined to be related to gender.

Of the 314 patients with LRV variations, 73 (23.2\%) had any sort of concomitant malignancy, the most common of which were gastrointestinal system malignancy, which appeared in 27 (37.0\%) cases and haematological malignancy which appeared in
Table 4. Cancers distribution according to location/system in patients with left renal vein variation

\begin{tabular}{lcc}
\hline & Number & Per cent of total \\
\hline Digestive/gastrointestinal & $\mathbf{2 7}$ & $\mathbf{3 7 . 0 \%}$ \\
Gastric cancer & 11 & $15.1 \%$ \\
Colon cancer & 7 & $9.6 \%$ \\
Pancreatic cancer & 6 & $8.2 \%$ \\
Rectal cancer & 2 & $2.7 \%$ \\
Oesophageal cancer & 1 & $1.4 \%$ \\
Haematologic/blood & $\mathbf{2 5}$ & $\mathbf{3 1 . 5} \%$ \\
Non-Hodgkin lymphoma & 9 & $12.3 \%$ \\
Hodgkin lymphoma & 7 & $9.6 \%$ \\
Haematologic malignancy & 7 & $9.6 \%$ \\
Others & & \\
Lung cancer & 4 & $5.5 \%$ \\
Genitourinary cancer & 4 & $5.5 \%$ \\
Gynaecologic cancer & 3 & $4.1 \%$ \\
Breast cancer & 3 & $4.1 \%$ \\
Miscellaneous & 9 & $12.3 \%$ \\
Total & $\mathbf{7 3}$ & $\mathbf{1 0 0 . 0 \%}$ \\
\hline
\end{tabular}

23 (31.5\%) cases (Table 4). There was no statistical difference in the retroaortic LRV and circumaortic LRV groups in terms of gender $(p=0.269)$ and malignancy frequency $(p=0.59)$.

\section{DISCUSSION}

The development of renal veins and IVC are highly complex processes associated with each other. This process covers the period between the fourth and eighth weeks of the embryogenesis, and consequently, IVC develops from a network of three parallel vascular pairs, including posterior cardinal, lower cardinal, and supracardinal vessels, in order of appearance. During this period, anastomotic communication occurs between the subcardinal and supracardinal canals with a vascular collar surrounding the anterior and posterior aorta. The dorsal arch of the circumaortic collar regresses and the progression 
of the ventral arch of the circumaortic collar forms a normal LRV. Conversely, the RLRV is caused by the persistence of the dorsal arch of the renal collar and the regression of the ventral arch, so that a single LRV passes through posterior to the aorta. CLRV results from the persistence of both the dorsal and ventral arches of the renal ring so that the LRVs pass through both anterior and posterior to the aorta $[2,14]$.

Although the clinical and imaging findings of the mesoaortic compression of the LRV (nutcracker phenomenon) are well described in the literature, there is limited information on RLRV and CLRV. With the increased use of imaging modalities, the identification of these variations has become clinically important for both surgical and diagnostic reasons. Recognition of vascular variations before renal venous sampling, preoperative evaluation of renal donors, splenorenal shunt placement, vena cava filter placement, nephrectomy, aortic surgery, and staging of renal cell carcinoma is crucial to preventing misdiagnosis and complications, such as retroperitoneal haemorrhage, nephrectomy, and even death. Recognition of these variations is also important in the differential diagnosis of retroperitoneal pathology [8-10].

Even though abdominal venous structures can be evaluated with different radiological modalities, such as CT, CT angiography, Doppler, magnetic resonance and ultrasonography, CT is the preferred imaging modality for the evaluation of abdominal venous vascular structures due to higher patient compliance, lower cost, fast and easy applicability and reliability $[1,3,4,7]$.

The prevalence of RLRV and CLRV was reported to be $0.5-7.4 \%$ and $0.3-6.3 \%$, respectively, in a newer various studies conducted by using autopsy series, $\mathrm{CT}, \mathrm{CT}$ angiography, magnetic resonance, and positron emission tomography-computed tomography $[1,3-7,9,10,13,16-19]$. The reported incidence of LRV variations varies widely (Table 3 ). The high difference between the study results may be due to the different modalities of the studies, patient characteristics included in studies or the number of patients. However, in general, the incidence of LRV variations is around $2 \%$.

Our study included the CT images of 12,341 patients which is one of the most comprehensive studies in the literature in terms of investigation of LRV variations. Similar to previous studies in the literature, the incidences of $L R V$, RLRV, and CLRV variations were also found to be $2.27 \%, 1.56 \%$, and $0.71 \%$, respectively, in our study. The results of our study show that the incidence of total LRV variations in the Turkish population is similar to that of other races $[9,13,15,17,19]$.

Similarly to our results, most of the studies reported that the incidence of RLRV variations was higher than that of CLRV variations [3, 5-7, 16-18]. However, some studies found that the incidence of CLRV variations higher that RLRV variations $[1,9,10,13]$. RLRV variations are less complex than CLRV variations. This may be due to different embryological development processes of these renal vein variations.

While there was no gender correlation regarding LRV in some previous studies, Dilli et al. found that RLRV was more common in females than in males $[5,6,18,19]$. In our study, there was no correlation between total LRV and CLRV; and gender. In contrast to Dilli et al. [5], we found that the incidence of RLRV was statistically significantly higher in males than in females. This difference between the studies may be due to differences in the number, characteristics of patients included in the studies or due to differences in investigation modalities.

Interestingly, there was a concomitant malignancy in $73(23.2 \%)$ of 314 cases with LRV variations in our study. The majority of concomitant malignancies were gastrointestinal system (37.0\%) or haematologic (31.5\%) malignancies. Of the 27 (37.0\%) concomitant gastrointestinal system malignancies, 11 (15.1\%) were gastric cancer, 7 (9.6\%) were colon cancer, $6(8.2 \%)$ were pancreatic cancer, $2(2.7 \%)$ were rectal cancer and $1(1.4 \%)$ was oesophageal cancer. Of the 25 (31.5\%) accompanying haematologic malignancies, 9 (12.3\%) were Hodgkin's lymphoma, 7 (9.6\%) were non-Hodgkin's lymphoma and 7 (9.6\%) were other haematologic malignancies (Table 4). There is no comprehensive information in the literature other than a few cases with left renal variation accompanied by malignancy $[11,12]$. More extensive studies are needed to demonstrate whether there is a correlation between the embryological development of the renal vein variation and malignancy development.

\section{Limitations of the study}

Our study has some limitations. Abdominal CT examinations of all the cases included in the study were performed for any reasons such as trauma scan, diagnosis of abdominal pathology, and cancer screening or follow-up, however, the results of the study cannot be generalised to the entire population since healthy people were not included in the study because 
of the radiation risk. Despite that, the size of the study population is the main strength of this study.

\section{CONCLUSIONS}

In conclusion, CT is a fast and reliable method for detecting renal vein variations. Of the LRV variations, RLRV variation is more common than CLRV variation. The presence of total variations in LRV and CLRV is not associated with gender; whereas the presence of RLRV variations is more common in males.

The incidence of LRV variation is above $2 \%$ and incidentally identified vascular variations during routine CT scans should always be reported since they are of clinical and surgical importance. During routine abdominal CT reporting, it should be kept in mind that LRV variation will be detected in approximately one of every 50 patients, and if not detected, the renal vascular structures should be evaluated more carefully.

Because of the high rate of malignancy detected in patients with LRV variations in our study, we suggest that patients with LRV variations in imaging modalities should be carefully evaluated especially for gastrointestinal and haematologic malignancies.

\section{REFERENCES}

1. Ayaz $S$, Ayaz ÜY. Detection of retroaortic left renal vein and circumaortic left renal vein by PET/CT images to avoid misdiagnosis and support possible surgical procedures. Hell J Nucl Med. 2016; 19(2): 135-139, doi: 10.1967/ s002449910367, indexed in Pubmed: 27331207.

2. Bass JE, Redwine MD, Kramer LA, et al. Spectrum of congenital anomalies of the inferior vena cava: cross-sectional imaging findings. Radiographics. 2000; 20(3): 639-652, doi: 10.1148/radiographics.20.3.g00ma09639, indexed in Pubmed: 10835118.

3. Boyaci N, Karakas E, Dokumacı DS, et al. Evaluation of left renal vein and inferior vena cava variations through routine abdominal multi-slice computed tomography. Folia Morphol. 2014; 73(2): 159-163, doi: 10.5603/ FM.2014.0017, indexed in Pubmed: 24902094.

4. Çınar C, Türkvatan A. Prevalence of renal vascular variations: Evaluation with MDCT angiography. Diagn Interv Imaging. 2016; 97(9): 891-897, doi: 10.1016/j. diii.2016.04.001, indexed in Pubmed: 27178758.

5. Dilli A, Ayaz UY, Kaplanoğlu $H$, et al. Evaluation of the left renal vein variations and inferior vena cava variations by means of helical computed tomography. Clin Imaging. 2013; 37(3): 530-535, doi: 10.1016/j.clinimag.2012.09.012, indexed in Pubmed: 23102929.

6. Dilli A, Ayaz UY, Karabacak OR, et al. Study of the left renal variations by means of magnetic resonance imaging. Surg
Radiol Anat. 2012; 34(3): 267-270, doi: 10.1007/s00276011-0833-7, indexed in Pubmed: 21618012.

7. Dilli A, Coşar Ayaz FS, Karacan K, et al. Evaluation of renal anomalies, inferior vena cava variations, and left renalvein variations by lumbar magnetic resonance imaging in 3000 patients. Turk J Med Sci. 2017; 47(6): 1866-1873, doi: 10.3906/sag-1611-23, indexed in Pubmed: 29306251.

8. Ghandour A, Partovi S, Karuppasamy K, et al. Congenital anomalies of the IVC-embryological perspective and clinical relevance. Cardiovasc Diagn Ther. 2016; 6(6): 482-492, doi: 10.21037/cdt.2016.11.18, indexed in Pubmed: 28123970.

9. Jee WC, Lee W, Yong HY, et al. CT angiography for living kidney donors: accuracy, cause of misinterpretation and prevalence of variation. Korean J Radiol. 2008; 9(4): 333-339, doi: 10.3348/kjr.2008.9.4.333, indexed in Pubmed: 18682671.

10. Koc Z, Ulusan S, Oguzkurt L, et al. Venous variants and anomalies on routine abdominal multi-detector row CT. Eur J Radiol. 2007; 61(2): 267-278, doi: 10.1016/j. ejrad.2006.09.008, indexed in Pubmed: 17049792.

11. Muller Arteaga C, Martín Martín S, Cortiñas González $J R$, et al. [Posterior nutcracker syndrome: retroaortic renal vein associated with arteriovenous fistula and renal cancinoma. Report of a case and review of literature]. Actas Urol Esp. 2009; 33(1): 101-104, doi: 10.1016/ s0210-4806(09)74012-4, indexed in Pubmed: 19462735.

12. Otsuki H, Kuroda K, Kosaka T, et al. A case report of retroaortic left renal vein with tumor thrombus of renal cell carcinoma. Clin Exp Nephrol. 2011; 15(3): 438-441, doi: 10.1007/s10157-011-0410-8, indexed in Pubmed: 21327697.

13. Reed MD, Friedman AC, Nealey P. Anomalies of the left renal vein: analysis of 433 CT scans. J Comput Assist Tomogr. 1982; 6(6): 1124-1126, doi: 10.1097/00004728198212000-00013, indexed in Pubmed: 7174929.

14. Sadler TW. Langman's medical embryology. Wolters Kluwer Health/Lippincott Williams \& Wilkins. 2011: 384.

15. Sahin C, Kaçira OK, Tüney D. The retroaortic left renal vein abnormalities in cross-sectional imaging. Folia Med (Plovdiv). 2014; 56(1): 38-42, doi: 10.2478/folmed-2014-0006, indexed in Pubmed: 24812921.

16. Satyapal KS, Kalideen JM, Haffejee AA, et al. Left renal vein variations. Surg Radiol Anat. 1999; 21(1): 77-81, doi: 10.1007/BF01635058, indexed in Pubmed: 10370998.

17. Sośnik $H$, Sośnik K. Renal vascularization anomalies in the Polish population. Pol Przegl Chir. 2017; 89(6): 26-30, doi: 10.5604/01.3001.0010.6760, indexed in Pubmed: 29335391.

18. Yeşildağ $A$, Adanır $E$, Köroğlu $M$, et al. Rutin abdomen BT'de sol renal ven anomalilerinin görülme sıklığı. Tanısal ve Girişimsel Radyoloji. 2004; 10: 140-3.

19. Zhu J, Zhang L, Yang Z, et al. Classification of the renal vein variations: a study with multidetector computed tomography. Surg Radiol Anat. 2015; 37(6): 667-675, doi: 10.1007/ s00276-014-1403-6, indexed in Pubmed: 25567101. 